\title{
Improving Assistance in Inclusive Educational Settings (IMAS II)
}

\section{Entwicklung und Evaluierung web-basierter Wissensboxen zur Förderung inklusiver Kompetenzen von SchulassistentInnen}

\section{Caroline Breyer \& Barbara Gasteiger-Klicpera}

Journal für Psychologie, 27(2), 29-49

https://doi.org/10.30820/0942-2285-2019-2-29

www.journal-fuer-psychologie.de

\section{Zusammenfassung}

Bei der Umsetzung eines inklusiven Bildungssystems nehmen SchulassistentInnen eine Schlüsselfunktion ein. Sie unterstützen Kinder und Jugendliche mit Behinderung beim Regelschulbesuch und tragen entscheidend zu einem erfolgreichen Bildungsweg dieser SchülerInnen bei. Obwohl ihr Tätigkeitsfeld umfassend ist, weisen Untersuchungen darauf hin, dass SchulassistentInnen sich selbst nicht als ausreichend qualifiziert einschätzen und es ihnen an spezifischen Kompetenzen mangelt, um Kinder mit Behinderungen zu unterstützen und Inklusion zu ermöglichen. Um diesem Qualifizierungsbedarf zu entsprechen, werden im Rahmen des internationalen Kooperationsprojekts IMAS II fünf web-basierte Wissensboxen zu Themen, die für den Berufsalltag von AssistentInnen bedeutsam sind, konzipiert. Die Wissensboxen werden gemeinsam mit AssistentInnen, den begleiteten Kindern und ihren MitschülerInnen als MitforscherInnen entwickelt, anschließend mit AssistentInnen, LehrerInnen, Eltern und SchülerInnen erprobt und evaluiert. Diese flexible Fortbildungsmöglichkeit soll zur Qualitätssteigerung und längerfristigen Qualitätsentwicklung der Schulassistenz sowie zum Gelingen von Inklusion im Schulbereich beitragen.

Schlüsselwörter: Schulassistenz, Qualifizierung, Professionalisierung, Inklusion, partizipative Forschung

\section{Summary}

Improving Assistance in Inclusive Educational Settings (IMAS II). Conception and Evaluation of web-based Knowledge Boxes to enhance inclusive competences of teaching assistants

Teaching assistants play a key role in the implementation of an inclusive educational system. They support children and adolescents with special educational needs (SEN) in regular 
schools and contribute in a decisive way to a successful education of these students. Although their field of activity is very comprehensive, scientific evidence indicates that assistants do not consider themselves to be sufficiently qualified and lack specific competences to support students with SEN and enable inclusion. In order to meet this need for qualification, five webbased knowledge boxes on topics relevant to the daily work of assistants are being designed and evaluated within the framework of an international cooperation project IMAS II funded by the European Union. The knowledge boxes will be developed together with assistants, accompanied students and their classmates as co-researchers and then tested with assistants, teachers, parents and students. The impact of the knowledge boxes will be evaluated. This flexible further training opportunity is intended to contribute to a quality improvement and long-term quality development of assistance services and to the success of inclusion in schools.

Key words: teaching assistants, inclusive education, participatory research, professional development, training

\section{$1 \quad$ Einleitung}

Mit der Ratifizierung der UN-Behindertenrechtskonvention haben sich die Mitgliedsstaaten zur Umsetzung eines inklusiven Bildungssystems verpflichtet (Artikel 24). Dies bedeutet, dass allen Kindern und Jugendlichen eine gleichberechtigte Teilhabe an Bildung ermöglicht wird, unabhängig von Geschlecht, sozio-ökonomischem Hintergrund sowie besonderen Lernbedürfnissen. Allerdings hat der Begriff der Inklusion sehr unterschiedliche Facetten und ermöglicht unterschiedliche Interpretationen (für eine Diskussion dieser Fragen siehe Grosche 2015). Inklusion wird als die Ermöglichung und Realisierung sozialer Partizipation betrachtet, als die Verwirklichung von Teilhabe und Anerkennung von Diversität. Die Wertschätzung von Verschiedenheit und die Ermöglichung einer Lernumgebung, die die unterschiedlichen Lernbedarfe aller Kinder berücksichtigt, wurde im deutschsprachigen Raum unter dem Stichwort der Pädagogik der Vielfalt diskutiert (Prengel 2006). Schulische Inklusion verfolgt das Ziel, die Fähigkeiten jedes Kindes und die daraus resultierenden pädagogischen Bedarfe anzuerkennen und jedes Kind seinen Fähigkeiten entsprechend individuell zu fördern, damit sich alle Kinder bestmöglich zu autonomen und mündigen Personen entwickeln können (Feyerer 2012). Diesem Verständnis von Inklusion folgt auch der vorliegende Artikel. Es erscheint klar, dass die Entwicklung eines inklusiven Bildungssystems Zeit braucht und nur ein Schritt nach dem anderen möglich ist (Ainscow 2005). Um diesem Anspruch gerecht zu werden und ein inklusives Bildungssystem umzusetzen, sind Veränderungen in der Schulstruktur und -organisation nötig, die die bestehenden Schulsysteme mit vielfältigen Herausforderungen konfrontieren (u. a. Laubner, Lindmeier und Lü- 
beck 2017, 7; Bernasconi und Böing 2017). Um schulische Inklusion zu ermöglichen, sind laut UN-Behindertenrechtskonvention innerhalb des Bildungssystems angemessene Vorkehrungen entsprechend den individuellen Bedürfnissen der SchülerInnen zu treffen und notwendige Unterstützungsleistungen anzubieten (United Nations 2006, n.p.). Eine dieser Unterstützungsleistungen kann die Begleitung durch SchulassistentInnen sein, die - wenn sie sorgfältig implementiert wird - als bedeutsamer Schritt für die Entwicklung inklusiver Bildungssysteme anzusehen ist (u. a. Fegert et al. 2016, 8; Henn et al. 2014, 398). Da es Schulen oftmals an Ressourcen zur Umsetzung von Inklusion mangelt, besteht die Aufgabe von Schulassistenz vor allem darin, die Kluft zwischen den Unterstützungsbedürfnissen der Kinder und dem, was Schulen derzeit an Unterstützung bieten können, zu überbrücken. Ohne die Unterstützung durch SchulassistentInnen wäre es vielen SchülerInnen nicht möglich, eine Regelschule zu besuchen (Laubner, Lindmeier und Lübeck 2017, 7).

\section{Schulassistenz als Unterstützung auf dem Weg zu einer inklusiven Schule}

SchulassistentInnen begleiten jene Kinder und Jugendliche im schulischen Alltag, »die auf Grund besonderer Bedürfnisse im Kontext Lernen, Verhalten, Kommunikation, medizinischer Versorgung und/oder Alltagsbewältigung der besonderen und individuellen Unterstützung bei der Verrichtung unterrichtlicher und außerunterrichtlicher Tätigkeiten bedürfen « (Dworschak 2010, 133-34). Obwohl diese Beschreibung relativ klar zu sein scheint, kann die Bezeichnung für diese Tätigkeit sehr unterschiedlich sein. In manchen Ländern wird von Schulassistenz gesprochen (Bacher, Pfaffenberger und Pöschko 2007), in anderen von Schulbegleitung (Laubner, Lindmeier und Lübeck 2017), Teaching Assistance (Webster et al. 2010) oder von Paraprofessionals (Giangreco, Suter und Doyle 2010). Gemeinsam ist diesen Maßnahmen, dass sie auf eine Unterstützung zur selbstbestimmten und vollen Teilhabe an der Gesellschaft und am gemeinsamen Unterricht abzielen (Feyerer, Prammer und Prammer-Semmler 2017, 6). Allerdings zeigen sich deutliche Unterschiede zwischen einzelnen Ländern in den gesetzlichen Bestimmungen und Grundlagen für die Schulassistenz. Während Schulassistenz in einigen Ländern als einzelfallorientierte Maßnahme zur Begleitung und Unterstützung von Kindern mit Behinderung in Regelschulen eingesetzt wird, die als Teil des Schulsystems verstanden wird (u. a. Giangreco und Doyle 2007, 430-31; Webster et al. 2010), ist Schulassistenz in anderen Ländern lediglich als externe zusätzliche Maßnahme konzipiert, die von den sozialen Diensten geleistet wird und weitgehend unabhängig vom Schulsystem ihrer Aufgabe nachkommt (u.a. Dworschak 2017, 37; Thiel 2017). In den österreichischen Gesetzmäßigkeiten zur Maßnahme Schulassis- 
tenz ist beispielsweise deutlich formuliert, dass SchulassistentInnen (ausschließlich) für pflegerisch-helfende Tätigkeiten zuständig sind, um Kinder mit Sonderpädagogischem Förderbedarf (SPF) im Unterricht zu unterstützen. Pädagogische Tätigkeiten liegen nicht in ihrem Aufgabenbereich (Rechtsinformationssystem des Bundes 2014, 5-6).

Trotz dieser rechtlichen Bestimmungen, die auch in anderen Ländern, wie Deutschland, Portugal oder Großbritannien, ähnlich formuliert sind, zeigen Untersuchungen sehr deutlich, dass die geforderte Abgrenzung zu pädagogisch-unterrichtlichen Tätigkeiten in der Praxis kaum haltbar ist. So gaben etwa in der Studie von Henn et al. (2014) 90\% der befragten SchulassistentInnen in Deutschland an, das begleitete Kind häufig oder zumindest manchmal im Unterricht praktisch zu unterstützen. Weitere 54.3\% der Befragten äußerten, die SchülerInnen häufig für die Teilnahme am Unterricht zu aktivieren und auch Unterstützung bei der Emotionsregulation zu leisten (Henn et al. 2014, 401). Vergleichbare Befunde wurden in der österreichischen Untersuchung von Bacher et al. (2007) berichtet, in der sich zeigte, dass SchulassistentInnen durchaus pädagogische Tätigkeiten in Form von Anleiten, Anpassen von Lerninhalten, Vorbereiten von Unterrichtsmaterialien, oder auch die Planung des Unterrichts übernahmen. Ferner fanden auch Webster et al. (2010) für Großbritannien, dass die befragten SchulassistentInnen in einem nicht unerheblichen Ausmaß in einer direkten pädagogischen Rolle mit dem Kind interagierten und es sowohl im Einzelsetting als auch in Kleingruppen unterstützten.

\section{Spannungsfelder und kritische Entwicklungstendenzen im Zusammenhang mit schulischer Assistenz}

Die Divergenz zwischen den eng gesetzten rechtlichen Bestimmungen zur Maßnahme Schulassistenz und den Aufgaben, die in der Praxis von den SchulassistentInnen wahrgenommen werden, stellt nur eines von mehreren Spannungsfeldern dar, in dem sich SchulassistentInnen bewegen. Ein weiterer schwieriger Bereich sind die unterschiedlichen Erwartungen seitens der Eltern und LehrerInnen, die an SchulassistentInnen herangetragen werden. Während Lehrkräfte von SchulassistentInnen erwarten, dass sie in ihrer Lehrtätigkeit sowie im Unterricht mit allen SchülerInnen unterstützt werden (Butt und Lowe 2012), äußerten Eltern hingegen den Wunsch, dass SchulassistentInnen ihr Kind im Rahmen einer Einzelförderung beim Lernen unterstützten (Peters 2015). Diese divergierenden Erwartungen sind unter anderem auf das Fehlen einer einheitlichen Rollen- und Aufgabenbeschreibung von AssistentInnen zurückzuführen (AFET - Bundesverband für Erziehungshilfe e.V. 2016, 4-5).

Zudem trägt die von der Schule unabhängige Organisation der Assistenzdienstleistung durch eigene soziale Dienstleistungsanbieter, wie etwa in Österreich und 
Deutschland, zu einem unklaren Rollen- und Aufgabenverständnis und damit verbundenen Unklarheiten in den jeweiligen Zuständigkeits- und Verantwortungsbereichen der beteiligten Professionen bei. SchulassistentInnen werden oft nicht als Teil des Schulkollegiums wahrgenommen und sind selten in das schulische Geschehen eingebunden, was eine gemeinsame Arbeitsplanung erschwert (AFET - Bundesverband für Erziehungshilfe e. V. 2016, 4).

Neben unklaren Aufgaben- und Zuständigkeitsbereichen gibt es in vielen Ländern, wie etwa Österreich und Deutschland, auch keine klar umrissenen Anforderungsprofile oder spezifische Aus- und Fortbildungsmaßnahmen für SchulassistentInnen. Eine Ausnahme bildet das im Auftrag der Baden-Württemberg Stiftung von Fegert und Ziegenhain am Universitätsklinikum Ulm entwickelte Curriculum »Schulbegleiter - Fortbildungskonzept für die Qualifizierung von Schulbegleiterinnen und Schulbegleitern « (Baden-Württemberg Stiftung 2019). Ein Qualifizierungsangebot erscheint dringend notwendig, da sich SchulassistentInnen selbst als nicht ausreichend qualifiziert einschätzen und es ihnen an Kompetenzen mangelt, um Kinder mit Behinderung zu unterstützen und Inklusion zu ermöglichen (Bacher, Pfaffenberger und Pöschko 2007). Durch den Einsatz von nicht (ausreichend) qualifizierten SchulassistentInnen laufen Schulen Gefahr, Fehleinschätzungen und -entscheidungen bei der Förderung von Kindern mit Behinderung zu treffen und sie in ihrer Entwicklung nicht bedarfsgerecht zu unterstützen (AFET - Bundesverband für Erziehungshilfe e. V. 2016, 5).

Die unzureichende Qualifikation von SchulassistentInnen könnte auch ein Grund für einen negativen Einfluss der SchulassistentInnen auf den Lernerfolg von SchülerInnen mit Behinderung sein, wie Webster und seine MitarbeiterInnen (2010) berichteten. Zudem konnten diese AutorInnen eine mit steigendem Behinderungsgrad des Kindes zunehmende Interaktion zwischen SchulassistentInnen und dem begleiteten Kind beobachten, während die Interaktion zwischen der Lehrkraft und dem Kind mit Behinderung abnahm (Webster et al. 2010).

Diese Befunde machen auf die Gefahr aufmerksam, dass die/der Schulassistent/in zur einzigen Ansprechperson für das Kind wird. Illustriert wird die besondere Rolle, die SchulassistentInnen für die begleiteten Kinder einnehmen, durch die Ergebnisse der Studie von Broer, Doyle und Giangreco (2005). Sie fragten Jugendliche mit intellektuellen Beeinträchtigungen nach ihrer Einschätzung über die SchulassistentInnen. Die Ergebnisse zeigten, dass die befragten Jugendlichen ihre Schulassistentin/ihren Schulassistenten als FreundIn, BeschützerIn und als hauptverantwortliche Lehrkraft wahrnahmen.

Die intensive Unterstützung durch die SchulassistentIn birgt auch die Gefahr, zur Unselbstständigkeit des Kindes beizutragen (Schulze 2017). Zudem kann die ständige Anwesenheit von AssistentInnen sowie die Fixierung auf ein spezifisches Kind auch eine hemmende Wirkung auf die soziale Entwicklung des begleiteten Kindes haben (Böing und Köpfer 2017). Das fehlende bzw. unklare Rollen- und Aufgabenverständnis 
schulischer Assistenz kann dazu beitragen, dass sich SchulassistentInnen zu sehr für das Kind, das sie begleiten, verantwortlich fühlen und dadurch eine zu enge Beziehung zu dem Kind aufbauen, die in der Folge die Selbstständigkeit des Kindes, den Kontakt zu den MitschülerInnen und damit auch die Inklusion in die Klassengemeinschaft hemmt. Schulze (2017) weist darauf hin, dass nicht nur ein unklares Rollen- und Aufgabenverständnis, sondern auch ein mangelndes Reflexionsvermögen sowie das Fehlen einer spezifischen Qualifikation ein ausbalanciertes Nähe-Distanz-Verhältnis beeinträchtigen (Schulze 2017, 104-05).

Zusammenfassend lässt sich festhalten, dass die Tätigkeit von SchulassistentInnen von einem unklaren Rollen- und Aufgabenverständnis gekennzeichnet ist und trotz hoher Anforderungen ohne entsprechende Qualifizierung ausgeübt wird (Webster et al. 2010; Geist 2017; Schulze 2017; Blasse 2017). Um der Notwendigkeit nach Professionalisierung von schulischer Assistenz zu entsprechen (AFET - Berufsverband für Erziehungshilfe e. V.2016, 4-5), zielt das durch die Europäische Union geförderte Projekt IMAS II (Improving Assistance in Inclusive Educational Settings II) darauf ab, SchulassistentInnen eine niederschwellige und adäquate Weiterbildungsmöglichkeit zu bieten.

\section{Web-basierte Wissensboxen als Beitrag zur Professionalisierung schulischer Assistenz}

Die Basis für das Projekt bilden aktuelle wissenschaftliche Befunde, die eine Notwendigkeit der Professionalisierung schulischer Assistenz deutlich machen (u. a. Webster et al. 2010; Bacher, Pfaffenberger und Pöschko 2007; Carter et al. 2009). Im Rahmen dieses Projekts werden fünf web-basierte Wissensboxen zu Themen, mit denen SchulassistentInnen am häufigsten in ihrem Berufsalltag konfrontiert sind, entwickelt, implementiert und evaluiert.

\subsection{Ziel der Wissensboxen}

Ziel der Wissensboxen ist es, SchulassistentInnen und weiterem interessierten pädagogischen Personal eine Möglichkeit zur selbstbestimmten, zeit- und ortsunabhängigen Weiterbildung zu bieten. Angeboten werden sowohl theoretisches Wissen zu spezifischen Störungsbildern und Behinderungsarten, als auch praktische Anregungen für den Umgang mit herausfordernden und kritischen Situationen, ebenso wie zur Gestaltung inklusiven Unterrichts. Die Wissensboxen mit den darin enthaltenen Materialien werden am Ende der Projektlaufzeit (2020) in fünf Sprachen vorliegen (Englisch, 
Deutsch, Bulgarisch, Portugiesisch und Slowakisch) und für alle interessierten Personen Open Access zugänglich sein. Damit sollen SchulassistentInnen weltweit für eine bedarfsgerechte Unterstützung von Kindern mit Behinderung qualifiziert, für schulische Inklusion sensibilisiert und in ihren inklusiven Kompetenzen für die praktische Arbeit mit Kindern gestärkt werden.

Um diese Zielsetzungen zu erreichen, wurde mit finanzieller Unterstützung der Europäischen Union und in Zusammenarbeit mit Universitäten aus Österreich (Universität Graz), Slowakei (Universität Trnava) und Portugal (Centre for Social Studies, Universität Coimbra), dem Europäischen Dachverband für Dienstleistungsorganisationen für Menschen mit Behinderung (EASPD), sozialen Dienstleistungsanbietern aus Österreich (Chance B), Bulgarien (Agency Vision), England (CSIE), Portugal (ARCIL) und der Slowakei (TENENET o. z.) und der aktiven Einbindung von erfahrenen SchulassistentInnen aus den Partnerländern im Sinne eines partizipativen Ko-Produktionsansatzes das Konzept der Wissensboxen entwickelt. Dieses Konzept umfasst theoretische Grundlagen, praktische Hinweise, Erfahrungsberichte, Interviews, etc. und die Inhalte werden derzeit von den sozialen Dienstleistungsanbietern gemeinsam mit den Universitäten entwickelt. Jeweils eine weitere Universität oder Organisation eines anderen Landes fungiert als Tandempartner und unterstützt durch Ideen und Feedback zu den erarbeiteten Inhalten. Nach der Rohfassung findet ein Feedbackprozess statt, an dem sich alle PartnerInnen beteiligen. Sobald alle Rohfassungen vorliegen, werden die Inhalte auf eine gemeinsame Plattform hochgeladen und reihum werden in einer Feedbackschleife Kommentare und Anregungen zu jeweils einer Wissensbox gegeben. Anschließend wird diese überarbeitet, dann erfolgt die Rückmeldung durch die/den nächste/n Partner/in. Dieser Prozess ermöglicht es, fundierte Rückmeldungen zu den Inhalten zu geben, die Materialien mit theoretischer und praktischer Expertise anzureichern und unterschiedliche transnationale Aspekte zu berücksichtigen. Im Folgenden werden zunächst die Themen der Wissensboxen begründet und anschließend wird die Konzeption näher beschrieben.

\subsection{Inhalte der Wissensboxen}

Untersuchungen zeigen, dass SchulassistentInnen häufig Kinder mit Autismus-Spektrum-Störungen (59,4\%), SchülerInnen mit Mehrfachbehinderungen (26,3\%), Kinder mit Aufmerksamkeitsdefizit-/Hyperaktivitätsstörungen (ADHS) (35,9\%) und mit Störungen des Sozialverhaltens (20\%), ebenso wie SchülerInnen mit einer geistigen Behinderung $(8,2 \%)$ im Rahmen ihres Schulbesuches unterstützen und begleiten (Leitenbauer und Wilfling 2018, 11; Fegert et al. 2016, 18; Hafner 2016, 65; Henn et al. 2014, 400). Auch die Erfahrungen aus dem Vorgängerprojekt IMAS I (EASPD 2017) belegen, dass in den Partnerländern die Betreuung von Kindern mit diesen Förderbedarfen von besonde- 
rer Wichtigkeit ist. Daher sind die Inhalte der Wissensboxen diesen Themen gewidmet. Zudem kommt das Bemühen der internationalen Projektpartnerschaft um eine möglichst inklusive Sprache auch in den Bezeichnungen der Wissensboxen zum Ausdruck. Eine Wissensbox wird sich mit dem Thema »Lernen und Kognition « auseinandersetzen. Inhaltlich wird ein Fokus auf Kinder mit intellektuellen Entwicklungsstörungen sowie Down-Syndrom gelegt. Eine weitere Wissensbox wird sich der Thematik »Interaktion und Kommunikation « widmen. Diese konzentriert sich auf Autismus-Spektrum Störungen. Die dritte Wissensbox wird das Thema »Verhalten « aufgreifen und einen Schwerpunkt auf herausforderndes Verhalten bei Kindern und Jugendlichen legen. In der vierten wird das Thema »Körper- und Sinnesbeeinträchtigungen « erörtert.

Die letzte Wissensbox beschäftigt sich mit »Einstellungen zu Inklusion und Wahrnehmung von Behinderung « sowie mit dem Rollenverständnis von SchulassistentInnen und mit der Gestaltung der Zusammenarbeit mit Lehrkräften, Eltern und weiterem pädagogischem Personal. Diese Wissensbox thematisiert grundlegende Aspekte der Arbeit von SchulassistentInnen. Es wird die Frage diskutiert, was Inklusion bedeutet, und wie Partizipation und Teilhabe in der Schule umgesetzt werden können (Werning und Lütje-Klose 2012). Die Inhalte sollen die SchulassistentInnen zur Auseinandersetzung mit ihrem eigenen Rollenverständnis ermutigen, dazu anregen, Einstellungen zu hinterfragen, Klarheit in den fehlenden Strukturen zu schaffen (Schulze 2017, 104) und das Rollen- und Aufgabenverständnis der beteiligten Professionen zu thematisieren (Blasse 2017, 115-16). In Abbildung 1 werden die Themen der Wissensboxen und die dafür (haupt-)verantwortlichen Partnerorganisationen, die sich durch umfassende Expertise in dem jeweiligen Bereich auszeichnen, dargestellt.
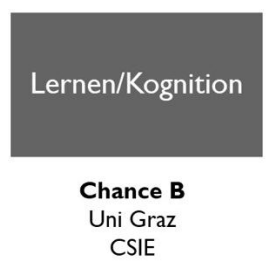

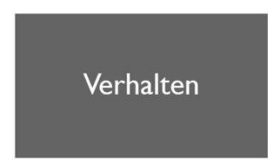

TENENET

Uni Trnava Uni Graz

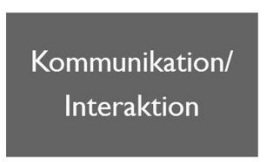

ARCIL CES Uni Graz
Körper- und Sinnes-

beeinträchtigungen

VISION

ARCIL

CSIE

\section{Einstellungen, Wahrnehmungen, Rollen}

\section{CSIE}

Chance B

Abbildung 1: Themen der Wissensboxen und (haupt-)verantwortliche Partnerorganisationen 


\subsection{Aufbau und Struktur der Wissensboxen}

Alle fünf Wissensboxen sind ähnlich aufgebaut. Jede Wissensbox enthält zunächst allgemeine Informationen zum jeweiligen Thema. Es werden theoretische Informationen zu Symptomen und Diagnostik gegeben, Ursachen beleuchtet und Möglichkeiten der Therapie und Förderung aufgezeigt. Dazu werden einführende Texte für ein Selbststudium zur Verfügung gestellt, Quizzes zur Einschätzung des eigenen Lernerfolgs, Videos zu diagnostischen Möglichkeiten sowie Interviews mit SchulassistentInnen, Lehrkräften, Eltern und SchülerInnen angeboten, um vielfältige Perspektiven einzubringen. Aufbauend auf den theoretischen Grundlagen umfasst jede Wissensbox konkrete praktische Handlungsansätze im Umgang mit kritischen und herausfordernden Situationen aus dem Schulalltag, die in Form von Videos, Erfahrungsberichten, Case-Studies und Good-Practice Beispielen dargestellt werden.

\subsection{Methodische Überlegungen zur Konzeption der Materialien}

Für jede Wissensbox werden Inhalte und Materialien ausgewählt, die theoretisch fundiert sind und deren Wirksamkeit im Rahmen empirischer Untersuchungen belegt wurde. Zusätzlich werden Materialien entwickelt, die die Expertise der PartnerInnen im Hinblick auf die ausgewählten Störungsbilder widerspiegeln. Es werden Videosequenzen produziert, in denen die Anwendung von Konzepten, wie etwa jenes der $\gg$ Augmentative and Alternative Communication $(\mathrm{AAC}) \ll$, gezeigt wird, deren Wirksamkeit empirisch belegt werden konnte, (u. a. Light und Mcnaughton 2015). Durch die Kooperation mit erfahrenen SchulassistentInnen ist es auch möglich, die Materialien mit praktischen Erfahrungen zu ergänzen und nutzerInnenfreundlich bereitzustellen.

Insgesamt wird bei der Entwicklung der Materialien darauf geachtet, praktisch relevante und theoretisch fundierte Inhalte zu konzipieren, die unterschiedlichen Ansprüchen und Anforderungen gerecht werden. Einerseits bedeutet das, dass die Inhalte und Materialien unterschiedliche Zielgruppen im Fokus haben, nämlich einerseits Grundschulkinder und andererseits auch Kinder der Sekundarstufe.

Zudem sollen die Materialien zur Wissensvermittlung und -erweiterung beitragen, indem sie an das unterschiedliche Vorwissen und die praktischen Erfahrungen der SchulassistentInnen in der Unterstützung von Kindern mit Behinderung anknüpfen. Um diesem Anspruch gerecht zu werden, wurden die Materialien zu theoretischen und diagnostischen Grundlagen in drei Komplexitätsniveaus entwickelt: ein Basislevel, ein ergänzendes Level und ein Vertiefungslevel. Das Basislevel bietet einen ersten Einblick in die Thematik, indem grundlegendes Wissen zu den jeweiligen Förderbedarfen ange- 
boten wird. Insgesamt ist für das Basislevel ein Zeitaufwand von etwa sechs Stunden pro Wissensbox vorgesehen.

Das zweite Level baut auf dem Basislevel auf und bietet vertiefende Inhalte. Beispielsweise finden sich auf dem zweiten Level weiterführende Informationen über Behinderungen unter Berücksichtigung der Sichtweisen unterschiedlicher Disziplinen (z.B. Medizin, Soziologie, Psychologie, Sonderpädagogik) oder aber umfangreichere Texte als Ergänzung zu den Textausschnitten des ersten Komplexitätslevels. Die dritte Ebene beinhaltet Forschungsartikel und weiterführende Texte, die Open Access zur Verfügung stehen, Links zu öffentlich zugänglichen Videos, Materialien oder Homepages von Organisationen. Diese Unterlagen dienen dazu, dass die SchulassistentInnen auch vertiefende Informationen erhalten, wenn sie dies wünschen. Die Aufbereitung der Inhalte in drei Komplexitätslevels bietet NutzerInnen die Möglichkeit, selbstbestimmt zu entscheiden, in welchem Ausmaß und in welcher Intensität sie sich mit den Inhalten der Wissensboxen auseinandersetzen wollen.

Darüber hinaus sollen auch die praktischen Inhalte der Wissensboxen den unterschiedlichen Erfahrungen gerecht werden, die SchulassistentInnen in der Unterstützung von Kindern mit Behinderung mitbringen. Die praktischen Beispiele greifen verschiedene Situationen im Umgang mit einem Kind mit Behinderung im Kontext Schule auf und thematisieren die Gestaltung gemeinsamen Lernens und die Förderung von Inklusion. Für SchulassistentInnen, die bislang nur wenig bis keine Erfahrung in der Begleitung von Kindern mit Behinderung haben, werden konkrete Anregungen gegeben, wie etwa zur Gestaltung des Erstkontakts mit dem Kind, den Eltern und den Lehrkräften, oder Tipps zum Beziehungsaufbau mit dem begleiteten Kind. Andererseits wird versucht, bereits erfahrenen SchulassistentInnen konkrete Anregungen und Handlungsansätze zum Umgang mit besonders schwierigen oder herausfordernden Situationen zu geben und ihre Handlungskompetenzen zu stärken.

Um diese konzeptionellen Überlegungen zu verdeutlichen, wird im Folgenden eine der fünf Wissensboxen genauer beschrieben und die Inhalte werden auszugsweise vorgestellt.

\subsection{Inhaltliche Schwerpunkte der Wissensboxen am Beispiel der Wissensbox »Lernen und Kognition«}

Die Wissensbox Lernen und Kognition gliedert sich in drei Teile rund um das Thema Lernen bei einer leichten intellektuellen Entwicklungsstörung (ICD-11: 6A00.0; World Health Organization 2018). Im ersten Teil werden Lernen und Lernprozesse allgemein besprochen, im zweiten Teil werden Schwierigkeiten im Lernen und im dritten Teil werden Fördermaßnahmen und inklusiver Unterricht thematisiert. Der erste Teil 
über Lernen und Lernprozesse hat das Ziel, eine theoretische Basis zu vermitteln. In Form von Cartoons wird ein Einstieg in die Thematik geboten, um SchulassistentInnen einen ersten Einblick in die Komplexität von Lernprozessen zu ermöglichen und zu einem besseren Verständnis sowie zu einer Sensibilisierung für die Vielfalt an Schwierigkeiten im Lernprozess beizutragen.

Im zweiten Teil werden Schwierigkeiten im Lernen dargestellt. Wie aus Abbildung 2 hervorgeht, werden zunächst Ursachen von Schwierigkeiten beim Lernen beleuchtet. Neben einer leichten intellektuellen Entwicklungsstörung wird auch das Down-Syndrom als mögliche Ursache für Schwierigkeiten beim Lernen aufgegriffen. Anhand von verschiedenen Definitionen und mithilfe der Beschreibung von Merkmalen, die für Personen mit einer intellektuellen Entwicklungsstörung sowie für Personen mit DownSyndrom charakteristisch sind, wird versucht, SchulassistentInnen einen Einblick in die entsprechenden Förderbedarfe zu geben (Schulze 2017) und zudem Klarheit in der Verwendung von Begrifflichkeiten zu schaffen. In diesem Zusammenhang soll auch die Abgrenzung einer leichten intellektuellen Entwicklungsstörung zu weiteren Störungsbildern thematisiert werden, die sich in ihren Diagnosekriterien und Fördermaßnahmen maßgeblich voneinander unterscheiden (Heimlich 2016). Die Basis dazu bieten internationale Klassifikationssysteme zur Diagnose von Krankheiten und psychischen Störungen (ICD-11: World Health Organization 2018; DSM-V: Falkai und Wittchen 2015).

Um Kinder mit Problemen beim Lernen zu unterstützen, ist eine förderdiagnostische Abklärung notwendig (Heimlich 2016, 130). Aus diesem Grund werden diagnostische Möglichkeiten, wie etwa gezielte Beobachtungen, Interviews mit Eltern und Lehrpersonen sowie Fragebögen besprochen. Mithilfe dieser diagnostischen Möglichkeiten, können SchulassistentInnen herausfinden, wo die Stärken und Schwächen eines Kindes liegen. Hierfür werden den SchulassistentInnen beispielsweise Anregungen zur Konstruktion von Situationen gegeben, in denen sie das Kind beobachten können. Zudem werden Beobachtungsbögen zur Verfügung gestellt, damit sie regelgeleitete Beobachtungen in unterschiedlichen Situationen des Schulalltages durchführen können. Schließlich werden Möglichkeiten der Interpretation dieser Beobachtungen besprochen und Folgerungen für die Förderung und Unterstützung der Kinder abgeleitet. Auf diese Weise werden SchulassistentInnen mit Handlungswissen und Methoden ausgestattet, die ihnen helfen, Schwierigkeiten von Kindern zu identifizieren und zu verstehen, um konkrete und individuell angepasste Unterstützungsmaßnahmen ableiten zu können (Heimlich 2016; Werning und Lütje-Klose 2012).

Die theoretischen Hinweise werden mit drei konkreten Fallbeispielen belegt. Diese begleiten die Lernenden durch die Wissensbox und zeigen spezifische Charakteristika von Kindern mit Schwierigkeiten im Lernen. Drei Kinder, bei denen eine leichte intellektuelle Entwicklungsstörung diagnostiziert wurde und die im Rahmen ihres 
Schulbesuchs durch eine/n Schulassistenten/in begleitet werden, stellen sich in kurzen Videosequenzen selbst vor und erzählen über sich. Um ein umfassenderes Bild zu zeichnen, werden die Kinder auch von ihren Eltern, ihren MitschülerInnen, einer Lehrkraft und von ihrer/m Schulassistenten/in in Form von kurzen Videos oder Texten beschrieben. Durch die Beschreibung der Kinder aus verschiedenen und zum Teil sehr persönlichen Perspektiven werden nicht nur verschiedene Facetten von Schwierigkeiten im Lernen sichtbar, sondern vor allem die Vielfalt an individuellen Stärken, Begabungen und Fähigkeiten dieser Kinder. Damit wird deutlich, dass kompetenzorientierte Sichtweisen die Grundlage für die gemeinsame Arbeit mit dem Kind darstellen. Auch Fördermöglichkeiten orientieren sich an den Kompetenzen des Kindes (Heimlich 2016). Gleichzeitig wird damit zum Ausdruck gebracht, wie vielfältig Kinder und ihre Fähigkeiten sind und dass diese Diversität als Chance für ein gemeinsames Lernen und als Bereicherung für den gemeinsamen Unterricht gesehen werden kann (Werning und Lütje-Klose 2012).

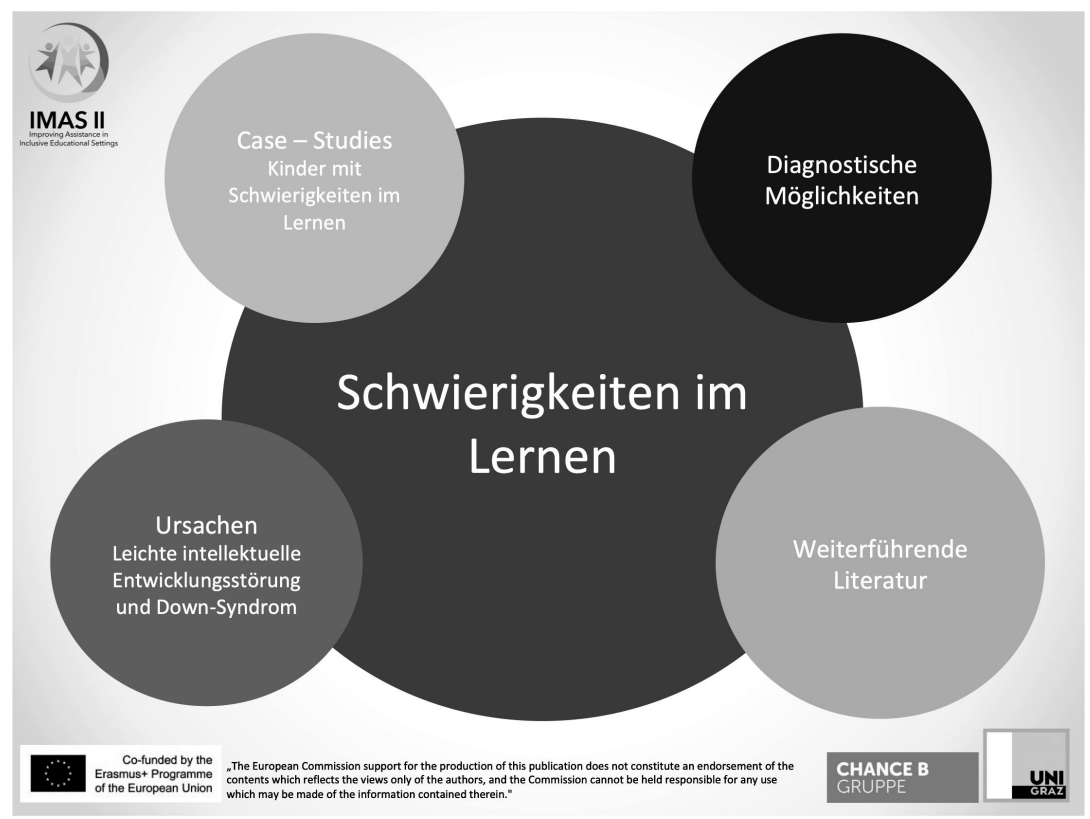

Abbildung 2: Gliederung des zweiten Schwerpunkts zum Thema Schwierigkeiten im Lernen

Im dritten Teil der Wissensbox Lernen und Kognition wird die Gestaltung inklusiven Unterrichts besprochen. Dieser Teil hat das Ziel, SchulassistentInnen die positive 
Bedeutung eines inklusiven Unterrichts für die individuelle und soziale Entwicklung von SchülerInnen näher zu bringen und die Gefahren von separierender Betreuung zu verdeutlichen (u. a. Sturm und Wagner-Willi 2018). Inhaltlich werden vielfältige praktische Anregungen und Ideen angeboten, um inklusiven Unterricht zu gestalten und das Kind in den gemeinsamen Unterricht einzubeziehen (Wilken 2008).

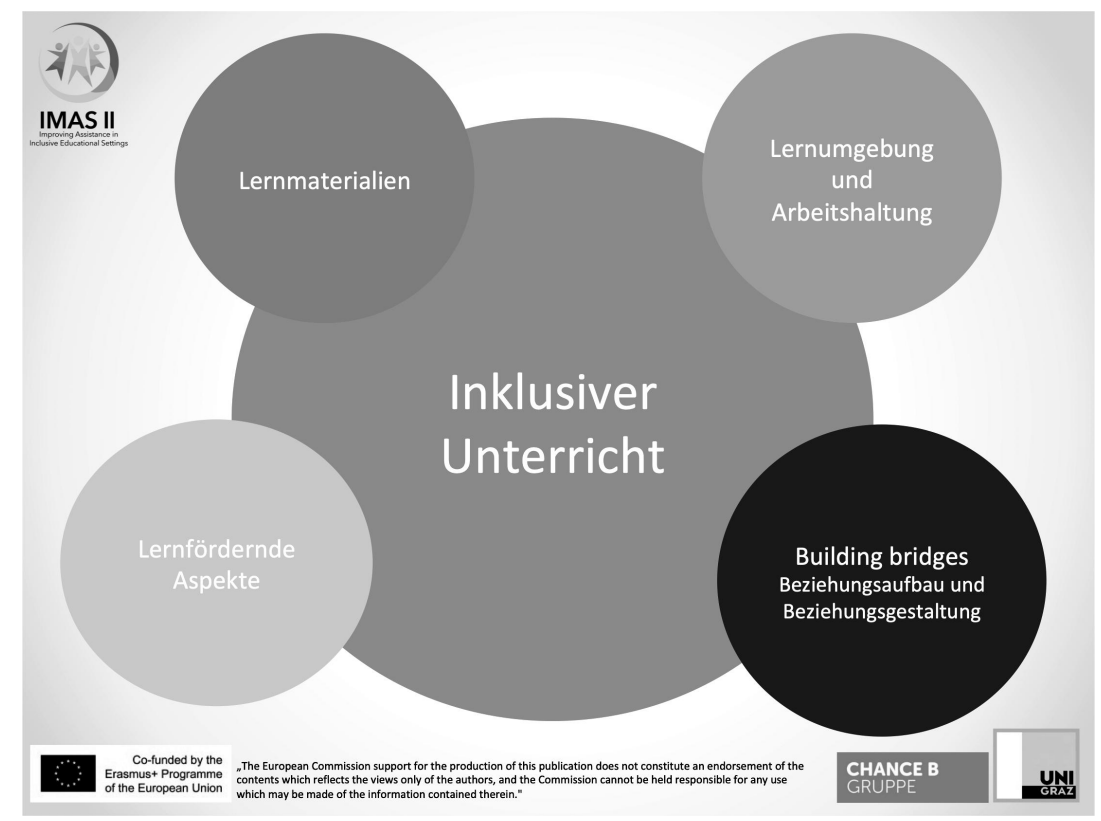

Abbildung 3: Gliederung des dritten Schwerpunktes zur Gestaltung eines inklusiven Unterrichts

Wie aus Abbildung 3 hervorgeht, werden in diesem Teil der Wissensbox zunächst Anregungen für die Gestaltung von Lernumgebungen und -arrangements gegeben, in denen sich Kinder wohlfühlen und in denen ein gemeinsames Lernen unter Einbezug der individuellen Lernvoraussetzungen der Kinder ermöglicht wird (Dobrowsky 2012; Werning und Lütje-Klose 2012; Mitchell 2008). Auch werden Ideen, wie Kinder zum Lernen ermuntert werden können, diskutiert (Hattie und Timperley 2007; Brophy 2013).

Da eine positive LehrerInnen-SchülerInnen-Interaktion das Lernen von SchülerInnen beeinflussen kann (Hattie 2008), werden Möglichkeiten zum Beziehungsaufbau sowie zur Beziehungsgestaltung zwischen SchulassistentInnen und dem begleiteten Kind aufgezeigt. Darüber hinaus wird auf die Beziehungsgestaltung mit Eltern, Lehrkräften und SonderpädagogInnen eingegangen. Zusätzlich werden den Schulassisten- 
tInnen Strategien zur Unterstützung von Interaktionen zwischen Kindern mit und ohne Behinderung erläutert (Causton-Theoharis und Malmgren 2005). Diese Themen werden in Form von kurzen Videosequenzen, Storyboards, Erfahrungsberichten, Texten und Good-Practice-Beispielen dargestellt.

Abschließend wird eine umfangreiche Sammlung an Methoden und Materialien zur Verfügung gestellt, mit deren Hilfe das Lernen von Kindern mit einer leichten intellektuellen Entwicklungsstörung unterstützt werden kann. Die Materialien sollen den SchulassistentInnen dabei helfen, den Unterrichtsstoff für die Kinder differenziert aufzubereiten. Mithilfe von konkreten Beispielen, die Situationen aus dem Schulalltag und spezifische Inhalte aus den Unterrichtsfächern Lesen, Schreiben und Mathematik aufgreifen, werden den SchulssistentInnen praktische Anregungen gegeben, damit die Kinder am gemeinsamen Unterricht teilnehmen und mit den anderen Kindern am selben Thema lernen können (Werning und Lütje-Klose 2012).

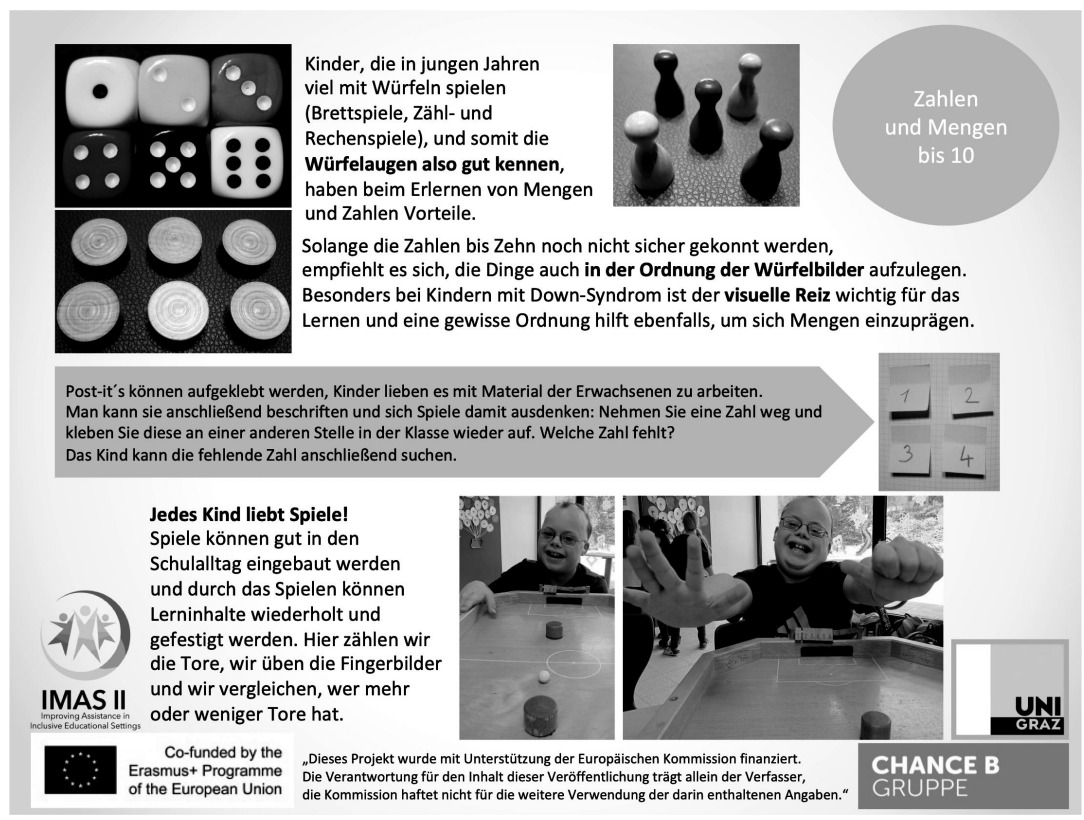

Abbildung 4: Beispiele für den Einsatz von Materialien und Methoden mit persönlichem Bezug im Mathematikunterricht, um das Zählenlernen zu unterstützen

Abbildung 4 zeigt exemplarisch Methoden, die die Teilnahme der Kinder am gemeinsamen Mathematikunterricht ermöglichen können. Um beispielsweise das Erarbeiten von Zahlenbegriffen anschaulicher zu machen, eigenen sich verschiedene visuelle Un- 
terstützungen, die in den Unterricht miteinbezogen werden (Wilken 2008, 29). Dazu können Materialien herangezogen werden, die in jeder Schule vorhanden sind. Passende Materialien können aber auch gemeinsam mit dem Kind erstellt und erarbeitet werden. Dadurch wird das Kind von Beginn an in den Prozess miteinbezogen, es kann sich aktiv einbringen und kreative Ideen beisteuern oder auch Entscheidungen über die Wahl und die Herstellung der Materialien treffen. Weitere Teilfertigkeiten, wie etwa die Feinmotorik beim Schneiden oder beim Legen kleiner Steine, werden gefördert. Neben dem Einsatz vielfältiger Materialien eignen sich auch Methoden, die sich durch einen persönlichen Bezug zum Kind auszeichnen. Wie in Abbildung 4 erkennbar, kann beispielsweise ein gemeinsames Tischfußballspiel dazu beitragen, Lerninhalte aus dem Mathematikunterricht zu wiederholen und zu festigen.

\subsection{Geplante Evaluierung}

Die Implementierung der Wissensboxen wird durch einen Evaluierungsprozess begleitet. Das Ziel der geplanten Evaluierung liegt darin, den Implementationsprozess wissenschaftlich zu begleiten und die Wirksamkeit der Wissensboxen zu untersuchen, um gegebenenfalls gezielte Veränderungen und Adaptierungen beim Aufbau und den Inhalten der Wissensboxen vornehmen zu können.

Dazu werden neben der aktiven Einbindung von SchulassistentInnen in die Konzeption und Erstellung der Wissensboxen, auch Kinder aus allen Partnerländern in die Evaluierung einbezogen. Im Rahmen eines partizipativen Forschungsansatzes werden Interviews und Fokusgruppen mit Kindern aus Schulen geführt, die sich durch eine hohe Diversität in den Klassen auszeichnen. Um für alle Kinder eine aktive Beteiligung an den geplanten Forschungsaktivitäten sicherzustellen, werden Kinder mit und ohne Behinderung, die gemeinsam eine Klasse besuchen, für ihre Aufgabe zu Ko-ForscherInnen ausgebildet. Ziel dieses Forschungsansatzes ist es, die begleiteten Kinder und ihre MitschülerInnen zu ihrer Sicht auf Schulassistenz, zu ihren Erfahrungen damit und zu ihren Wünschen zu befragen. Gemeinsam mit den SchülerInnen soll erforscht werden, wie SchulassistentInnen einen Beitrag zur Entwicklung eines inklusiven Bildungssystems leisten können und welche Unterstützung seitens der AssistentInnen für die Kinder wichtig oder hilfreich ist. Für die Ausbildung der SchülerInnen zu Ko-ForscherInnen wird auf bereits erprobte Konzepte zurückgegriffen (Messiou 2013). Dabei erwerben Kinder Kompetenzen der Beobachtung, führen selbst Fotodokumentationen, diskutieren in Fokusgruppen zu Fragen des gemeinsamen Lernens und zu Möglichkeit der Unterstützung durch SchulassistentInnen. Die Ergebnisse der partizipativen Forschung mit den Kindern werden anschließend in die Weiterentwicklung der Wissensboxen einfließen. 
Darüber hinaus wird im Anschluss an die erste Pilotphase, in der SchulassistentInnen die Wissensboxen nutzen, das neu erworbene Wissen im Zusammenhang mit den inklusiven Kompetenzen von SchulassistentInnen aus unterschiedlichen Perspektiven evaluiert. Dazu werden Lehrkräfte, Eltern und SchulassistentInnen aus allen Partnerländern mithilfe von Fragebögen und Reflexionsgesprächen zu ihrer Einschätzung der Effekte der Wissensboxen auf die Kompetenzen von SchulassistentInnen befragt. Der Fragebogen wird von einer der Partneruniversitäten konstruiert und setzt sich aus Items zusammen, die auf die Zielsetzungen der Inhalte aus den Wissensboxen abgestimmt sind. Zusätzlich werden bestehende Skalen, wie etwa zur Einstellung zu Inklusion (Hellmich und Görel 2014) oder zur Arbeit im Team (Kauffeld 2004), einbezogen. Insgesamt soll eine Stichprobe von 500 Personen zu ihrer Einschätzung der Effekte der Wissensboxen befragt werden. Es wird erwartet, dass sich durch die Nutzung der Wissensboxen das Wissen von SchulassistentInnen zur Unterstützung von Kindern mit Behinderungen erweitert und sich ihre Handlungsmöglichkeiten und ihre inklusiven Kompetenzen verbessern. Zur Einschätzung durch Lehrkräfte wird erwartet, dass sich eine Verbesserung in der Zusammenarbeit zwischen LehrerInnen und SchulassistentInnen zeigt und diese im Umgang mit SchülerInnen als kompetenter eingeschätzt werden. Schließlich wird erwartet, dass auch Eltern die SchulassistentInnen im Umgang mit den SchülerInnen als kompetenter einschätzen. Nachdem die Evaluierungsergebnisse vorliegen, erfolgt eine gemeinsame Reflexion mit SchulassistentInnen, Lehrkräften und Eltern, um die Inhalte der Wissensboxen zu adaptieren und anzupassen.

Mit der geplanten Evaluierung soll sichergestellt werden, dass die Inhalte der Wissensboxen das Ziel einer nutzerInnenfreundlichen Weiterbildungsmöglichkeit erreichen und dass sie zur Qualitätsentwicklung der Maßnahme Schulassistenz beitragen.

\section{Zusammenfassung}

Auf dem Weg zu einem inklusiven Bildungssystem nehmen SchulassistentInnen eine Schlüsselfunktion ein. Sie begleiten und unterstützen Kinder und Jugendliche mit unterschiedlichen Behinderungen bei ihrem Schulbesuch und tragen damit entscheidend zu einem erfolgreichen Bildungsweg dieser SchülerInnen bei (u.a Laubner, Lindmeier und Lübeck 2017). Aktuelle Forschungsbefunde zeigen jedoch, dass sich SchulassistentInnen selbst nicht als ausreichend qualifiziert einschätzen und es ihnen an Kompetenzen mangelt, um ihre vielfältigen Aufgaben zu bewältigen und um Inklusion zu ermöglichen (Bacher, Pfaffenberger und Pöschko 2007). Gleichzeitig weisen Untersuchungen auch auf kritische Entwicklungstendenzen (u. a. Webster et al. 2010) und Spannungsfelder hin, in denen sich SchulassistentInnen befinden. Diese kritischen As- 
pekte verdeutlichen die Notwendigkeit einer Professionalisierung schulischer Assistenz und zeigen einen dringenden Qualifizierungsbedarf von SchulassistentInnen (Schulze 2017; Webster et al. 2010; Carter et al. 2009).

Um SchulassistentInnen auf ihre Aufgaben besser vorzubereiten, werden im Rahmen des durch die Europäische Union geförderten Kooperationsprojektes IMAS II fünf web-basierte Wissensboxen entwickelt und evaluiert, die dann eine leicht zugängliche und niederschwellige Weiterbildungsmöglichkeit für SchulassistentInnen darstellen. Die Wissensboxen greifen Themen aus dem beruflichen Alltag von SchulassistentInnen auf und beinhalten verschiedene Schwerpunkte. Zunächst werden inklusive Haltungen und Einstellungen thematisiert, wobei allerdings eher Fragen von Inklusion, Chancengleichheit und Teilhabe aufgeworfen werden, ohne Vorgaben und Wertungen. Gebündelt in vier weiteren Themen (Lernen und Kognition, Verhalten, Interaktion und Kommunikation, Körper- und Sinnesbeeinträchtigungen) werden theoretische Grundlagen zu Behinderung vermittelt, es werden Symptome und Ursachen beleuchtet sowie Therapien und Fördermöglichkeiten besprochen.

Um die SchulassistentInnen auf ihre täglichen Aufgaben vorzubereiten, werden in den Wissensboxen konkrete Handlungsansätze für den Umgang mit herausfordernden Situationen sowie zur Gestaltung inklusiven Unterrichts vorgestellt, die ein gemeinsames Lernen von Kindern mit und ohne Behinderung ermöglichen sollen. Darüber hinaus werden Lösungsansätze für die unterschiedlichen Spannungsfelder, mit denen SchulassistentInnen konfrontiert werden, thematisiert. Dazu gehören Anregungen zur Beziehungsgestaltung sowie Strategien zum Aufbau eines ausbalancierten Nähe-Distanz-Verhältnisses mit dem begleiteten Kind (Schulze 2017). Um die soziale Entwicklung der Kinder zu unterstützen, enthalten die Wissensboxen Anregungen zur Förderung von Peer-Interaktionen sowie zur Inklusion des begleiteten Kindes in die Klassengemeinschaft (Böing und Köpfer 2017). Auch kritische Aspekte der Wahrnehmung verschiedener Rollen im Team und der Zusammenarbeit mit Lehrpersonen werden thematisiert. Schließlich werden Anregungen für die multiprofessionelle $\mathrm{Zu}$ sammenarbeit sowie für die Zusammenarbeit mit Eltern gegeben, die als elementare Faktoren für gelingende Inklusion angesehen werden (Meyer 2017).

Mit der Entwicklung der web-basierten Wissensboxen wird versucht, der internationalen Forderung nach Professionalisierung schulischer Assistenz gerecht zu werden, SchulassistentInnen für die Anliegen und Schwierigkeiten schulischer Inklusion zu sensibilisieren, sie für die differenzierte Unterstützung von Kindern mit Behinderung zu qualifizieren, und in ihren inklusiven Kompetenzen für die praktische Arbeit mit Kindern zu stärken. Darüber hinaus wird durch die Evaluierung der Wissensboxen sichergestellt, dass diese für SchulassistentInnen gut verständlich und hilfreich sind, damit sie zu einer nachhaltigen Qualitätsentwicklung der Maßnahme Schulassistenz beitragen und das Gelingen schulischer Inklusion unterstützen können. 


\section{Literatur}

AFET - Bundesverband für Erziehungshilfe e. V. 2016. »Dokumentation des ExpertInnengesprächs zu aktuellen rechtlichen und fachlichen Spannungsfeldern bei der Schulbegleitung in Regelschulen.«Hannover. http://www.afet-ev.de/aktuell/AFET_intern/PDF-intern/2015/Expertengespr -Schulbegl-Nov15/01a.Dokumentation_Expertengesprch_04.11.2015.pdf?M = 1488976164.

Ainscow, Mel. 2005. »Understanding the development of inclusive education system." Electronic Journal of Research in Educational Psychology 3(3).

Bacher, Johann, Monika Pfaffenberger und Heidemarie Pöschko. 2007. Arbeitssituation und Weiterbildungsbedarfvon Schulassistent/Innen. Hagenberg: Pfaffenberger und Pöschko Sozialforschung.

Baden-Württemberg Stiftung. 2019. „Schulbegleiter«. https://www.bwstiftung.de/schulbegleiter/\# c20532

Bernasconi, Tobias, und Ursula Böing. 2017. »Einleitung.« In Inklusive Schulen Entwickeln. Impulse für die Praxis, herausgegeben von Tobias Bernasconi und Ursula Böing, 7-14. Bad Heilbrunn: Klinkhardt.

Blasse, Nina. 2017. „Vielfältige Positionen von Schulbegleitungen im Unterricht. «In Schulbegleitung in der inklusiven Schule. Grundlagen und Praxishilfen, herausgegeben von Marian Laubner, Bettina Lindmeier und Anika Lübeck, 107-17. Weinheim: Beltz.

Böing, Ursula, und Andreas Köpfer. 2017. "Schulassistenz aus der Sicht von Schülerinnen und Schülern mit Assistenzerfahrung." In Schulbegleitung in der Inklusiven Schule. Grundlagen und Praxishilfen, herausgegeben von Marian Laubner, Bettina Lindmeier und Anika Lübeck, 127-136. Weinheim: Beltz.

Broer, Stephen M., Mary Beth Doyle und Michael F. Giangreco. 2005. »Perspectives of Students With Intellectual Disabilities About Their Experiences With Paraprofessional Support.« Exceptional Children 71 (4): 415-30.

Brophy, Jere E. 2013. Motivating Students to Learn. 3. Aufl. New York: Routledge.

Butt, Rosemary, und Kaye Lowe. 2012. „Teaching Assistants and Class Teachers: Differing Perceptions, Role Confusion and the Benefits of Skills-Based Training." International Journal of Inclusive Education 16 (2): 207-2019. https://doi.org/10.1080/13603111003739678.

Carter, Erik, Laura O’ Rourke, Lynn G. Sisco und Danielle Pelsue. 2009. »Knowledge, Responsibilities, and Training Needs of Paraprofessionals in Elementary and Secondary Schools." Remedial and Special Education 30 (6): 344-59.

Causton-Theoharis, Julie, und Kimber Malmgren. 2005. »Building Bridges: Strategies to Help Paraprofessionals Promote Peer Interaction. «TEACHING Exceptional Children 37 (6): 18-24.

Dobrowsky, Angelika. 2012. „Den Raum umräumen. Die Schule als gestalteter Lern- und Lebensraum. «In Lernen und Raum. Gebaute Pädagogik und pädagogische Baustellen, herausgegeben von Erwin Rauscher, 155-64. Wolkersdorf: Paul Gerin.

Dworschak, Wolfgang. 2010. »Schulbegleiter, Integrationshelfer, Schulassistent. Begriffliche Klärung einer Maßnahme zur Integration in die Allgemeine Schule bzw. die Förderschule.» Teilhabe 3 (49): 131-35.

Dworschak, Wolfgang. 2017. „Zur Gewährung von Schulbegleitung - Wer erhält in welchem Umfang eine Schulbegleitung?« In Schulbegleitung in der inklusiven Schule. Grundlagen und Praxishilfen, herausgegeben von Marian Laubner, Bettina Lindmeier und Anika Lübeck, 37-49. Weinheim Basel: Beltz.

EASPD. 2017. »IMAS - Improving Assistance in Inclusive Educational Settings.« 2017. http://www. easpd.eu/en/content/imas.

Falkai, Peter, und Hans-Ulrich Wittchen. 2015. Diagnostisches und statistisches Manual psychischer Störungen DSM-5. Herausgegeben von American Psychiatric Association. Göttingen: Hogrefe.

Fegert, Jörg M, Ute Ziegenhain, Lydia Schönecker und Thomas Meysen. 2016. Schulbegleitung als 
Beitrag zur Inklusion. Schriftenreihe der Baden-Württemberg Stiftung Gesellschaft und Kultur Nr. 81. Stuttgart: Baden-Württemberg Stiftung.

Feyerer, Ewald. 2012. »Allgemeine Qualitätskriterien inklusiver Pädagogik und Didaktik.« Zeitschrift für Inklusion 6 (3). https://www.inklusion-online.net/index.php/inklusion-online/article/view/51/51. Feyerer, Ewald, Wilfried Prammer und Eva Prammer-Semmler. 2017. »Vorwort.« In Inklusion Konkret. Assistenz und Bildung, herausgegeben von Ewald Feyerer, Wilfried Prammer und Eva Prammer-Semmler, Band 2, 6-8. Linz: BZIB.

Geist, Eva-Maria. 2017. „Qualifikation und Qualifizierung von Schulbegleiter/innen.« In Schulbegleitung in der inklusiven Schule. Grundlagen und Praxishilfen, herausgegeben von Marian Laubner, Bettina Lindmeier und Anika Lübeck, 50-65. Weinheim Basel: Beltz.

Giangreco, Michael F, und Mary Beth Doyle. 2007. »Teacher Assistants in Inclusive Schools."In The SAGE Handbook of Special Education, herausgegeben von Lani Florian, 429-439 M4-Citavi. London: Sage.

Giangreco, Michael F, Jesse C Suter und Mary Beth Doyle. 2010. „Paraprofessionals in Inclusive Schools: A Review of Recent Research." Journal of Educational and Psychological Consultation 20: 41-57. https://doi.org/10.1080/10474410903535356.

Grosche, Michael. 2015. „Was Ist Inklusion? Ein Diskussions- und Positionsartikel Zur Definition von Inklusion aus Sicht der empirischen Bildungsforschung. «In Inklusion von Schülerinnen und Schülern mit Sonderpädagogischem Förderbedarf in Schulleistungserhebungen, herausgegeben von Poldi Kuhl, Petra Stanat, Birgit Lütje-Klose, Cornelia Gresch, Hans Anand Pant und Manfred Prenzel, 17-39. Wiesbaden: Springer VS.

Hafner, Katharina. 2016. „Vor- und Nachteile der Dienstleistung `Schulassistenz steirischen Schulassistent/-innen. Unveröffentlichte Masterarbeit: Universität Graz.

Hattie, John. 2008. Visible Learning. A Synthesis of Over 800 Meta-Analyses Relating to Achievement. London: Routledge. https://doi.org/10.4324/9780203887332.

Hattie, John, und Helen Timperley. 2007. »The Power of Feedback.« Review of Educational Research 77 (1): 81-112. https://doi.org/10.3102/003465430298487.

Heimlich, Ulrich. 2016. Pädagogik bei Lernschwierigkeiten. Sonderpädagogische Förderung im Förderschwerpunkt Lernen. 2. überarb. Ausgabe. Bad Heilbrunn: Verlag Julius Klinkhardt.

Hellmich, Frank, und Gamze Görel. 2014. »Erklärungsfaktoren für Einstellungen von Lehrerinnen und Lehrern zum inklusiven Unterricht in der Grundschule.« Zeitschrift für Bildungsforschung 4 (3): 227-40. https://doi.org/10.1007/s35834-014-0102-z.

Henn, Katharina, Leonore Thurn, Tanja Besier, Anne K Künster, Jörg M Fegert und Ute Ziegenhain. 2014. »Schulbegleiter als Unterstützung von Inklusion im Schulwesen. Zeitschrift für Kinderund Jugendpsychiatrie und Psychotherapie 42 (6): 397-403.

Kauffeld, Simone. 2004. FAT - Fragebogen zur Arbeit im Team. Göttingen: Hogrefe.

Laubner, Marian, Bettina Lindmeier und Anika Lübeck. 2017. "Schulbegleitung in der inklusiven Schule: Einführung in das Herausgeberwerk." In Schulbegleitung in der inklusiven Schule. Grundlagen und Praxishilfen, herausgegeben von Marian Laubner, Bettina Lindmeier und Anika Lübeck, 7-10. Weinheim Basel: Beltz.

Leitenbauer, Christoph, und Katharina Wilfling. 2018. "Qualifikationen von steirischen Schulassistent/innen - Besteht eine Notwendigkeit zur Professionalisierung des Berufsfeldes Schulassistenz?« Unveröffentlichte Masterarbeit: Universität Graz.

Light, Janice, und David Mcnaughton. 2015. „Designing AAC Research and Intervention to Improve Outcomes for Individuals with Complex Communication Needs Designing AAC Research and Intervention to Improve Outcomes for Individuals with Complex Communication Needs." Augmentative and Alternative Communication 31 (2): 85-96. https://doi.org/10. 3109/07434618.2015.1036458. 
Messiou, Kyriaki. 2013. »Working with Students as Co-Researchers in Schools.« International Journal of Inclusive Education 18 (6): 601-13. https://doi.org/10.1080/13603116.2013.802028 T4.

Meyer, Karina. 2017. »Multiprofessionalität in der inklusiven Schule: Eine empirische Studie zur Kooperation von Lehrkräften und Schulbegleiter/innen (Göttinger Schulbegleitungsstudie GötS).« Göttinger Beiträge zur erziehungswissenschaftlichen Forschung 37. https://doi.org/ https://doi.org/10.17875/gup2017-1029.

Mitchell, David. 2008. What Really Works in Special and Inclusive Education. Using Evidence-Based Teaching Strategies. London: Routledge.

Peters, Susanne. 2015. »Inklusive Bildung für Kinder mit komplexen Beeinträchtigungen? Elternperspektiven auf Schule.« PhD diss,. Universität Koblenz-Landau.

Prengel, Annedore. 2006. Pädagogik der Vielfalt. Verschiedenheit und Gleichberechtigung in interkultureller, feministischer und integrativer Pädagogik. 3. Auflage. Wiesbaden: VS Verlag für Sozialwissenschaften.

Rechtsinformationssystem des Bundes. 2014. Änderung Des Steiermärkischen Behindertengesetzes. https://www.ris.bka.gv.at/Dokumente/LgblAuth/LGBLA_ST_20140801_94/LGBLA_ST_ 20140801_94.html

Schulze, Kathrin. 2017. „Schulbegleitung im Spannungsfeld von Nähe und Distanz - Eine Einzelfallanalyse zum Umgang mit paradoxen Strukturen pädagogischen Handelns. «In Schulbegleitung in der inklusiven Schule. Grundlagen und Praxishilfen, herausgegeben von Marian Laubner, Bettina Lindmeier und Anika Lübeck, 97-106. Weinheim Basel: Beltz.

Sturm, Tanja, und Monika Wagner-Willi, herausgegeben 2018. Handbuch schulische Inklusion. Opladen: Verlag Barbara Budrich.

Thiel, Sylvia. 2017. »Die Beantragung und Bewilligung von Schulassistenz.« In Schulbegleitung in der inklusiven Schule. Grundlagen und Praxishilfen, herausgegeben von Marian Laubner, Bettina Lindmeier und Anika Lübeck, 28-36. Weinheim Basel: Beltz.

United Nations. 2006. „Convention on the Rights of Persons with Disabilities and Optional Protocol.« 2006. http://www.un.org/disabilities/documents/convention/convoptprot-e.pdf.

Webster, Rob, Peter Blatchford, Paul Bassett, Penelope Brown, Clare Martin und Anthony Russell. 2010. »Double Standards and First Principles: Framing Teaching Assistant Support for Pupils with Special Educational Needs. European Journal of Special Needs Education 25 (4): 319-36.

Werning, Rolf, und Birgit Lütje-Klose. 2012. Einführung in die Pädagogik bei Lernbeeinträchtigungen. München: Ernst Reinhardt Verlag.

Wilken, Etta. 2008. »Kinder, Jugendliche und Erwachsene mit Down-Syndrom.«In Sonderpädagogik der Geistigen Entwicklung, herausgegeben von Susanne Nußbeck, Adrienne Biermann und Heidemarie Adam, 18-34. Göttingen: Hogrefe.

World Health Organization. 2018. »|CD-11 for Mortality and Morbidity Statistics.« 2018. https://icd. who.int/browse11/I-m/en\#/http\%3A\%2F\%2Fid.who.int\%2Ficd\%2Fentity\%2F334423054.

\section{Die Autorinnnen}

Caroline Breyer, Bakk. phil., MSc, ist Doktorandin am Institut für Pädagogische Professionalisierung, Arbeitsbereich für Integrationspädagogik und Heilpädagogische Psychologie, der Universität Graz. Ihre Arbeitsschwerpunkte sind: Schulassistenz, Inklusion und Professionalisierung schulischer Assistenz.

Kontakt: caroline.breyer@uni-graz.at 
Barbara Gasteiger-Klicpera, Univ.-Prof. ${ }^{\text {in }}$ Dr.in, ist Professorin und Leiterin des Arbeitsbereichs für Integrationspädagogik und Heilpädagogische Psychologie am Institut für Pädagogische Professionalisierung der Universität Graz. Ihre Arbeitsschwerpunkte sind: Inklusive Bildung, Interventionen bei Leseschwierigkeiten, Differenzierter Unterricht, Kritische Gesundheitskompetenz und Evaluationsforschung.

Kontakt: barbara.gasteiger@uni-graz.at 